\title{
Continuum approach to computational multi-scale modeling of fracture
}

\author{
J. Oliver ${ }^{1, a}$, M. Caicedo ${ }^{1, b}$, E. Roubin ${ }^{1, c}$ and A.E. Huespe ${ }^{1,2, d}$ \\ ${ }^{1}$ Technical University of Catalonia (UPC)/International Center for Numerical Methods in \\ Engineering (CIMNE), Campus Nord UPC, Edifici C-1, 08034 Barcelona, Spain \\ ${ }^{2}$ CIMEC-Universidad del Litoral (UNL), Santa Fe, Argentina \\ axavier.oliver@upc.edu, ${ }^{\mathrm{b}} \mathrm{mcaicedo@cimne.upc.edu,}{ }^{\mathrm{c}}$ eroubin@cimne.upc.edu, \\ dahuespe@intec.unl.edu.ar
}

\begin{abstract}
Keywords: Fracture, computational multi-scale modeling, strain injection, crack path field, Continuum Strong Discontinuity Approach
\end{abstract}

\begin{abstract}
This paper presents a $\mathrm{FE}^{2}$ multi-scale framework for numerical modeling of the structural failure of heterogeneous quasi-brittle materials. The model is assessed by application to cementitious materials. Using the Continuum Strong Discontinuity Approach (CSD), innovative numerical tools, such as strain injection and crack path field techniques, provide a robust, and mesh-size, mesh-bias and RVE-size objective, procedure to model crack onset and propagation at the macro-scale.
\end{abstract}

\section{Introduction}

In view of the significant impact of low-scale material morphology in regards to macroscopic responses, numerical strategies has been wildly developed in order to both analyze and model the complex relationship between scales. Among the different issues that yet remain to be explored, this paper focuses on softening behavior modeling, i.e. non-smooth behaviors, and complex small scale failure mechanisms. These problematic find their applications, inter alia, in multi-scale modeling of the quasi-brittle fracture of cementitious materials.

The theoretical framework used in this work is based on the so-called ( $\left.\mathrm{FE}^{2}\right)$ methods. A coupled FEM is used in both scales. Homogenized quantities at the lower scale, represented by a so-called failure-cell, are therefore transferred, in a one-way fashion, to material points (Gauss points) of the macroscopic structure. Such a direct homogenization procedure eventually links this failure-cell to the notion of statistical Representative Volume Element (hereafter referred to as RVE). However, in case of softening behavior, the non-smooth kinematical description of the thin scale raises the problematic of existence of an RVE and the lack of objectivity of the response with respect to its size. Solutions have been provided, for example, in the context of regularized non-local damage models [1], or in the context of homogenized discrete models [4].

In contrast, this work is an attempt to address this issue in the context of local and continuum models and the Continuum Strong Discontinuity Approach (hereafter referred to as CSDA) [2]. The different key ingredients of the theoretical and numerical framework, which unlock the different issues and flaws mentioned above are: a) at the macroscopic level, the non-smooth kinematics representation is captured using strain-localization and FE with embedded regularized strong discontinuities, b) at the mesoscopic level, the failure-cell fracture behavior is fitted with cohesivebands with predefined position, even though there is no "a priori" limitation on the considered failure mechanisms, c) from the standard homogenization at the RVE the usual set of macroscopic quantities, in terms of a strain/stress relationship, emerges, together with, and specifically for this approach, a physically meaningful internal length linked with the size of the RVE and the activated microscopic fracture mechanism. This internal length is exported to the upper scale and used as the macroscopic strain localization bandwidth, this providing both an objective regularization parameter ---leading to a macroscopic response completely insensitive to RVE size--- and a consistent up-scaling scheme of the failure material properties ---such as the fracture energy. 


\section{Model description}

Mesoscopic scale description and homogenization procedure. The dissipative processes that occur at the mesoscale are modeled using cohesive bands (that are much thinner than the cell dimensions) scattered within the matrix, the aggregates and the interfaces between them. In this way, they can model a set of predefined crack patterns including several mechanisms such as percolation of the crack through the matrix (necessary for softening behavior), mortar/aggregate decohesion and rupture within the aggregates. Eventually, as the damage stage increases, a dominant mechanism naturally prevails, thus representing the final pattern of the micro-crack. This mechanism is now referred to as mesoscopic failure mechanism.

The meso-structure constitutive behavior is considered elastic outside these cohesive bands, whereas the material exhibits strain softening inside the bands (using a classical isotropic damage model), thus, eventually leading to strain localization. From the CSDA [2] it is well known that this kind of narrow bands represents, in a consistent way, a strong discontinuity in which the regularized parameter is given by its bandwidth, ensuring the equivalence of the fracture energy in both scales.

The approach is based on a first order homogenization of strains under a small strain framework in both scales, ensuring energetic balance through the Mandel-Hill principle). Though the mathematical framework is not developed here, attention is focused on the outcome of this homogenization procedure within the overall framework of the strong discontinuity kinematics. The macroscopic constitutive response is point-wise equivalent to an inelastic law (in an incremental fashion) as a function of the homogenized elastic tangent tensor, $C^{\text {hom }}$, and the incremental homogenized inelastic strain rate $\dot{\boldsymbol{\varepsilon}}^{(i)}$ i.e.

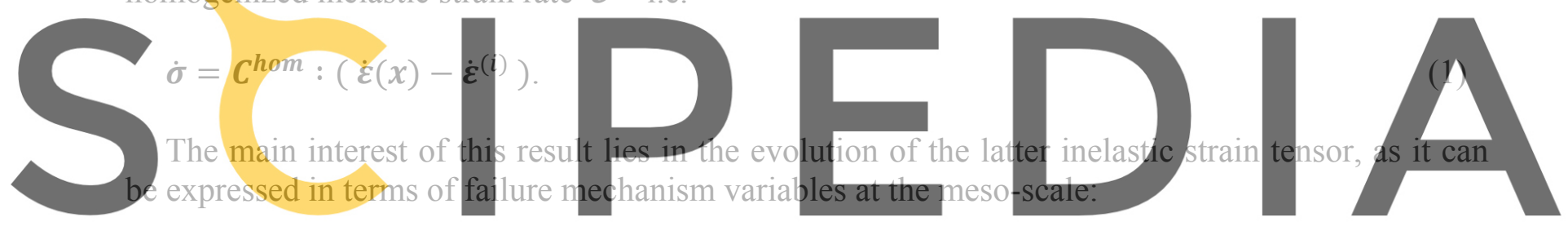

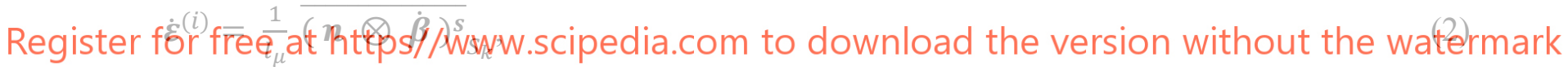

where the barked notation represents the average value the symmetrical tensor product between the strong discontinuity normal, $\boldsymbol{n}$, and the rate of the displacement jump, $\dot{\boldsymbol{\beta}}$, of each cohesive band, belonging to the manifold of the mesoscopic failure mechanism $S_{k}$, i.e. the mesoscopic crack. In addition, and defined as the ration between the measure (volume or area) of the RVE and the measure (surface or length) of $S_{k}, l_{\mu}$ stands the aforementioned material internal length.

Numerical aspects at the meso-cell. The cohesive bands are modeled by quadrilateral elements of very small thickness. Even though more general phenomenological models can be set, herein a constitutive only tension constitutive model [2] governs the softening behavior in order, first, to represent the typical tensile failure mechanisms of cementitious materials and second, to exhibit the structural impact of the heterogeneities that can lead to complex macroscopic responses.

The external force for the equilibrium equation is the macroscopic strain tensor since, consistently with the considered homogenization theory, no external force comes into play. Furthermore, and following the usual minimal boundary restrictions at the RVE, Dirichlet boundary conditions, avoiding rigid body motions, are applied.

Finally, it is worth noticing that the mesoscopic failure mechanism propagates naturally through the strain localization that takes place within the cohesive bands. At each time step of the problem, the in-loading cohesive bands define the active failure mechanism and therefore they define the set of elements used to upscale non-linear features such as the characteristic length of Eq. (2).

Material failure propagation at the structural level. The strain injection technique [5] is used in order to provide a robust and efficient model that can capture failure propagation even with high 
strain localization states. Two specific strain-rate modes (weak and strong discontinuity modes) are injected within two evolving sets of elements, following criteria based on up-scaled non-linear features, such as bifurcation analysis of the homogenized localization tensor, or based on the dissipated energy. In addition, the crucial matter of positioning strong discontinuities is tackled by a parallel technique termed crack path field [5]. This technique uses a directional derivative of a scalar field, based on the internal strain-like variables, whose zero level set defines the crack path.

Based on physical considerations of the crack characteristics ---both in terms of path and kinematics---, the coupling of these methods leads to a numerical framework capable of drastically reducing the classical drawbacks of propagating fracture approaches, such as stress locking, hourglass modes, mesh bias, etc. More details can be found, in a one-scale framework in [5].

\section{Numerical results: application to classical concrete-like simulations}

Mesoscopic material properties. The description of the meso-cell is taken to be the same for both tests. The FE discretization is depicted in Fig. 1a where the morphology and the considered pattern of cohesive bands can be seen (from dark to light gray: elastic aggregates, elastic mortar matrix and cohesive bands). The material elastic properties are taken to be the same for elastic and cohesive band elements.
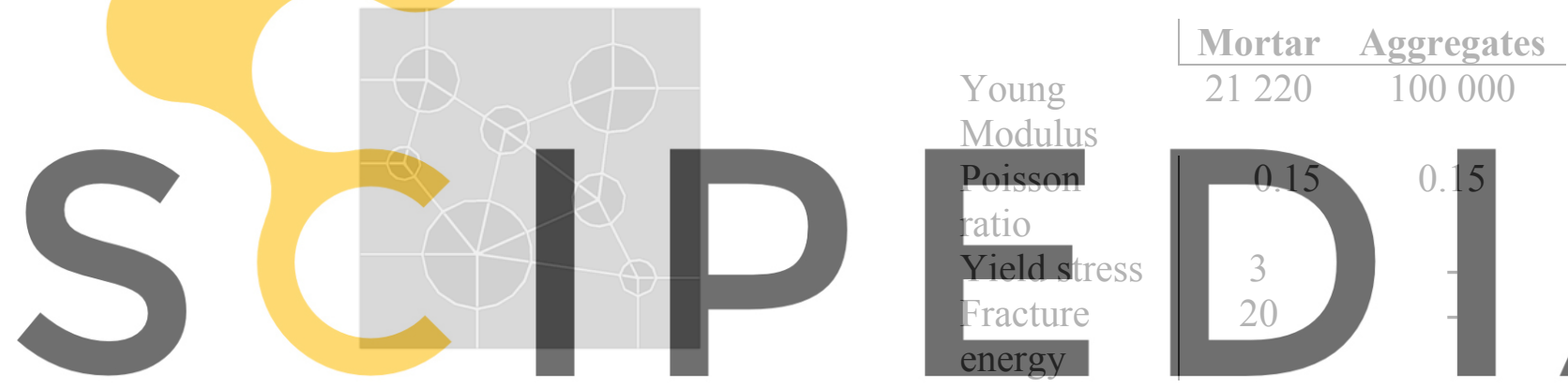

\section{$[\mathrm{MPa}]$}

Fig. 1. Mesoscopic FE discretization and considered material properties

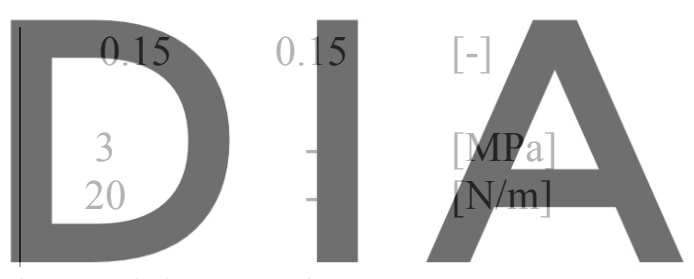

Register for free at https//www scipedia.com to download the yersion without the watermark prior to a tension loading, the specimen is subjected to shear load up to $5 \mathrm{kN}$ and $10 \mathrm{kN}$. First, a typical mesoscopic failure mechanism is depicted in Fig. 2a, where the activated cohesive bands are clearly shown. Figs. $2 b$ and $2 c$ represent the injection state (light gray for weak and dark gray for strong injection) for two different time steps, thus showing its evolving aspect. By enhancing the kinematics only in a narrow zone around the strain localization, the displacement discontinuity can be well capture and yet, instable modes (hourglass) are avoided. It can be checked that the injection pattern follows the crack path field shown Fig. 2 d.

a)

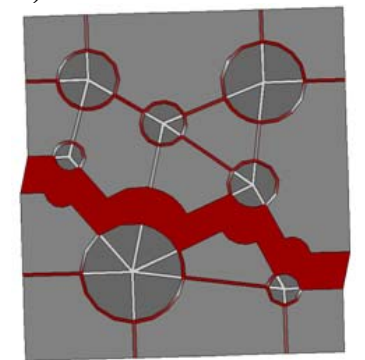

b)

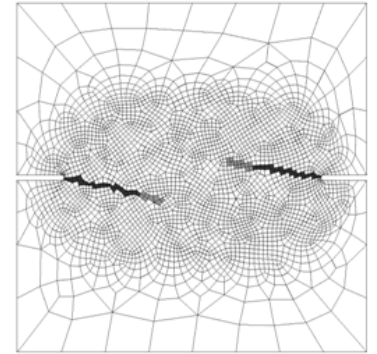

c)

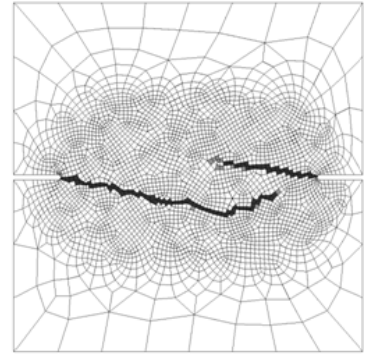

d)

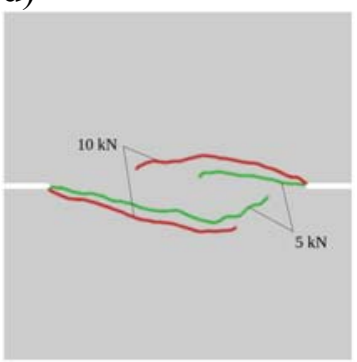

Fig. 2a: a) Damage in cell, b) Strain-injection pattern $(5 \mathrm{kN})$, c) Strain-injection pattern $(10 \mathrm{kN})$ and d) Crack path fields for $5 \mathrm{kN}$ and $10 \mathrm{kN}$.

The latter figure also represents the crack path field in the case of a shear loading of $10 \mathrm{kN}$. Matching the results of [3], a larger spacing between the two cracks along with a larger curvature can be noticed. 
Four point bending test. This example shows the impact of the meso-cell failure mechanisms onto the macroscopic response. The meso-cell (Fig. 1) has been slightly modified in order to perform four different tests whose results are depicted in Figs. 3a and 3b, in terms of the crack path field and the macroscopic responses, respectively. The first two meso-cells (top left and top right in Fig. 3a) allow and preclude, respectively, intra-aggregate failure. Therefore, they could correspond to typical failure mechanisms for hard and light-aggregate concrete. The other two tests (bottom right and bottom left) have the cohesive bands locked for failure excepting one horizontal and one vertical path, respectively, therefore restricting the set of possible mesoscopic failure mechanisms to a predetermined one. In Fig. 3a, the link of the mesoscopic failure mechanisms and the obtained macroscopic crack path can clearly be observed. Furthermore, in Fig. 3b, the translation of the considered failure-mechanisms into structural responses can be observed.

a)

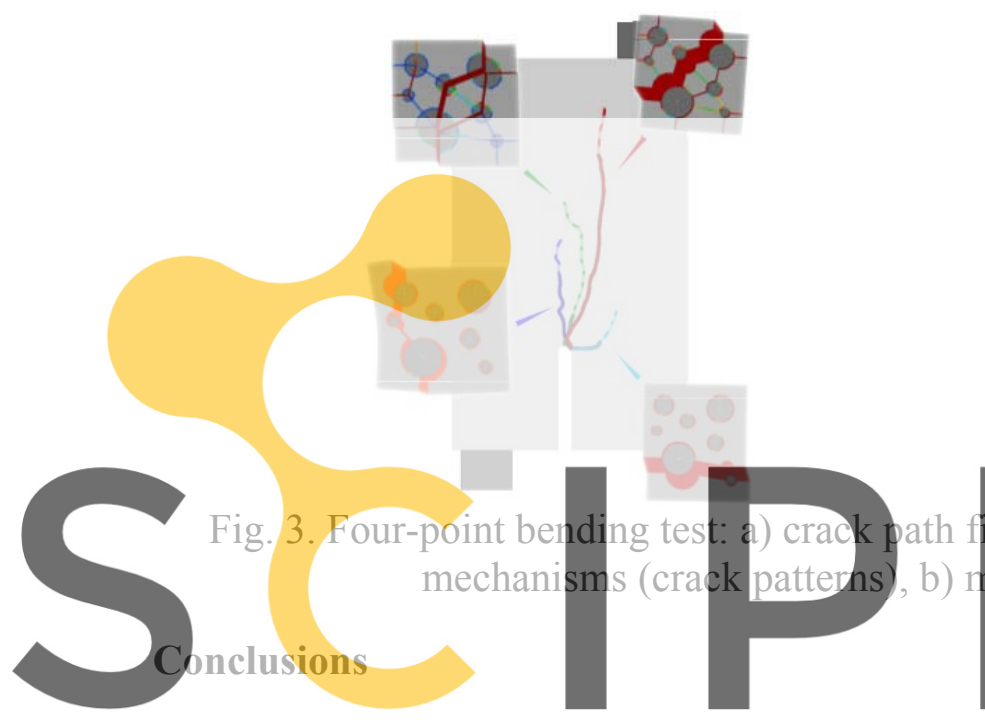

b)

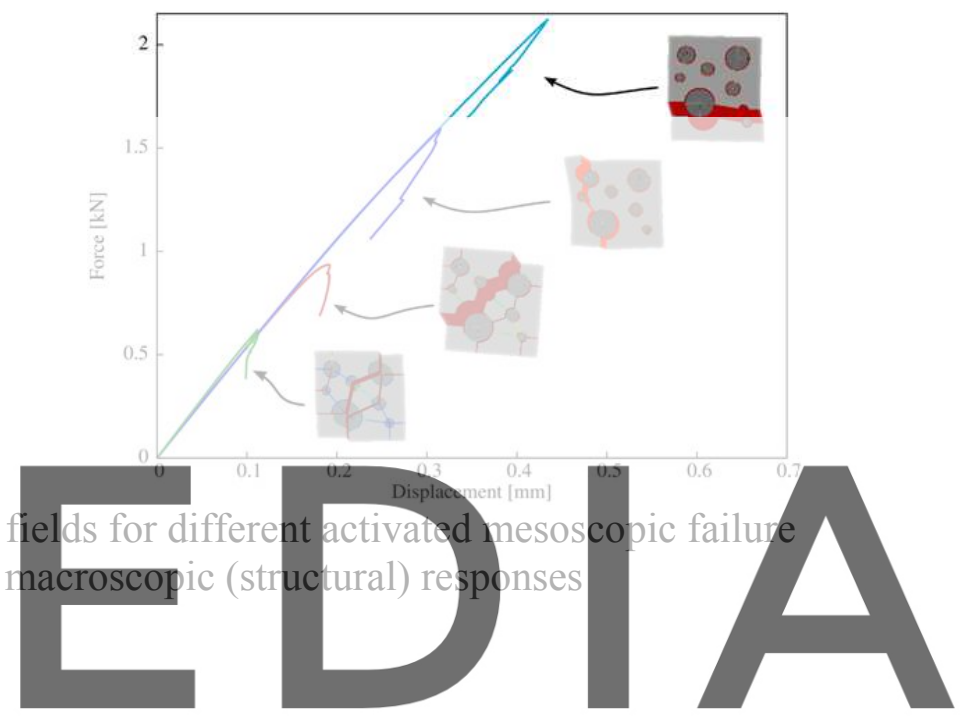

The result of this work is a multi-scale framework that preserves the correct dissipation and

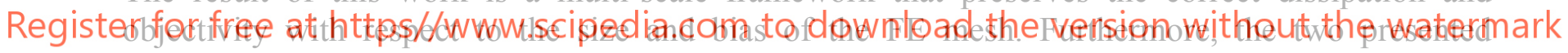
simulations show the, physically meaningful, effects of the activated meso-scale failure mechanisms on the structural response. The proposed framework allows extending the classical FE ${ }^{2}$ computational homogenization procedures from smooth problems to fracture mechanics analyzes.

\section{Acknowledgments}

The research leading these results has received funding from the European Research Council under the European Union's Seventh Framework Program (FP/2007-2013) / ERC Grant Agreement n. 320815, Advanced Grant Project COMP-DES-MAT. The third author would also like to thanks the École Normale Supérieur de Cachan (FR) for their financial support.

\section{References}

[1] V.P. Nguyen, O. Lloberas-Valls, M. Stroeven and L. J. Sluys, in: Computer Methods in Applied Mechanics and Engineering, (2010)

[2] J. Oliver, A.E. Huespe, M.D.G Pulido and E. Chaves, in: Engineering Fracture Mechanics, 2002

[3] M.B Nooru-Mohamed, E. Schlangen and J.G.M. Van Mier, in: Cement Based Materials, 1993

[4] P.J. Sánchez, P.J. Blanco, A.E. Huespe, and R.A. Feijóo in: Comp. Meth. In Appl. Mech. And Eng., 2013.

[5] J. Oliver, A. Huespe and I.F. Dias, in: Comp. Meth. In Appl. Mech. And Eng., 2014 